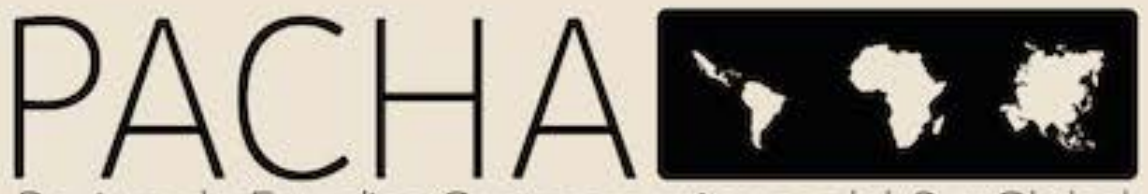

Revista de Estudios Contemporáneos del Sur Global Joumal of Contemporary Studies of the Global South Revista de Estudos Contemporâneos do Sul Global 


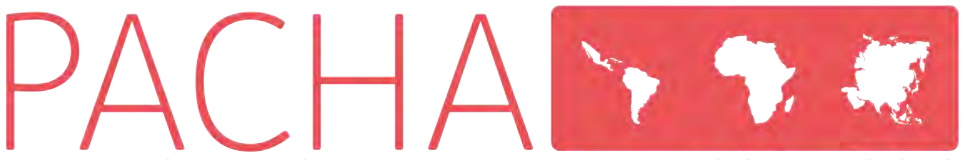

Revista de Estudios Contemporáneos del Sur Global

Joumal of Contemporary Studies of the Global South

\section{Equipo Editorial}

Carolina Díaz R.

Directora Editorial

CICSH-AL Centro de Investigaciones en Ciencias Sociales y Humanidades desde América Latina, Ecuador revistapacha@religacion.com; carolinadiaz@religacion.com

Roberto Simbaña Q.

Coordinador Editorial

CICSH-AL Centro de Investigaciones en Ciencias Sociales y Humanidades desde América Latina, Ecuador robertosimbana@religacion.com

\section{Editores Asociados}

Paola Andrea Tovar. Universidad De Montreal, Colombia. Editora Asociada en Antropología

Mitchell Alberto Alarcón Díaz. Universidad Nacional Mayor de San Marcos, Perú. Editor Asociado en Educación

Marcela Cristina Quinteros. Universidade Estadual de Maringá, Brasil. Editora Asociada en Historia Latinoamericana

Aboutaleb Sedatee Shamir. Islamic Azad University, Iran. Editor Asociado en Educación y Medio Oriente

Mateus Gamba Torres. Universidade de Brasilia - UNB, Brasil. Editor Asociado en Historia Mirna Yazmin Estrella Vega. Universidad Nacional Autónoma de México, México. Editora Asociada en Sociología

Rodrigo Navarrete Saavedra. Universidad Aus tral de Chile, Chile. Editor Asociado en Ciencias Políticas

Aygul Zufarovna Ibatova. Tyumen Industria University, Rusia. Editora Asociada en Humahidades sobre Asia
Shokhrud Fayziev Farmanovich. Tadqiqot, Tashkent, Uzbekistan. Editor Asociado en Desarrollo sobre la Comunidad de Estados Independientes, Asia.

Mª Aránzazu Serantes López. Woolf University, España. Editora Asociada en Humanidades digitales

Fabricio Espinosa Ortiz. Consejo Nacional de Ciencia y Tecnología, México. Editor Asociado en Geografía Humana

Marcelo Starcenbaum. Universidad Nacional de La Plata, Argentina. Editor Asociado en Filosofía e Historiografía

Siti Mistima Maat. Universiti Kebangsaan Malaysia, Malaysia. Editora Asociada en Innovación de aprendizaje

Carla Vanessa Zapata Toapanta. Universidad de Salamanca, España. Editora Asociada en Latinoamericanismo

\section{Consejo Editorial}

Andrea Paola Cantarelli, Universidad Nacional de Cuyo, Argentina
Federico Cabrera, Universidad Nacional de San Juan/CONICET, Argentina

Gamaniel David Suárez Cobix, Universidad Veracruzana, México

Gloria Concepción Tenorio Sepúlveda, Tecnológico de Estudios Superiores de Chalco, México

Héctor García Cornejo, Universidad Michoacana de San Nicolás de Hidalgo, México

Jorge Gilberto Bonilla Macas, Universidad Católica de Cuenca, Ecuador

José Alexander Rubiano Pedroza, Universidad de Pamplona, Colombia

María Dolores Sancho, Universidad Nacional del Comahue, Argentina

Mauricio Sandoval Cordero, Facultad Latinoamericana de Ciencias Sociales, Costa Rica

Rivera Varela Bertha Leticia, Universidad Abierta a Distancia, México

Tomás Sebastián Torres López, Universidad Alberto Hurtado, Chile

\section{CONSEJO ASESOR INTERNACIONAL}

\author{
Alejandro Mejía Tarazona \\ Facultad Latinoamericana de Ciencias Sociales, Ecuador \\ Alexander Luna Nieto \\ Fundación Universitaria de Popayán, Colombia \\ Celeste De Marco \\ CONICET/Universidad Nacional de Quilmes-CEAR, Argentina \\ Christian Andres Quinteros Flores \\ Universidad de Chile, Chile \\ Daniel Orizaga Doguim \\ Centro de Investigaciones Multidisciplinarias-UAQ, México \\ Francisco Javier Jover Mart \\ Universidad de Castilla-La Mancha, España
}

Gaya Makaran

Centro de Investigaciones sobre América Latina y el Caribe CIALC, UNAM, México

Jaime Araujo Frias

Universidad Nacional de San Agustín, Perú

João Luis Binde

Universidade Federal De Pernambuco, Brasil

Luisina Castelli Rodríguez

Universidad de la República, Uruguay

Mariana Jesica Lerchund

Universidad Nacional de Río Cuarto, Argentina

Marina Acosta

Universidad de Buenos Aires, Argentina

Noelia Marina Cortinas

Universidad de Buenos Aires, Argentin

Paulo Alves Pereira Júnior

Universidad Estatal Paulista, Brasil

Sergio Monroy Isaza

Universidad de Ibagué, Colombia

Suyai Malen Garcia Gualda

IPEHCS-CONICET-UNCO, Argentin 


\section{Cultura de Género en las Universidades en el marco de la socioformación y el desarrollo social sostenible: Análisis Documental}

Culture of Gender in the Universities in the framework of socioformation and sustainable social development: Documentary Analysis

Ana María Hernández-López

Universidad Autónoma de la Ciudad de México - México

ana.maria.hernandez@uacm.edu.mx

Josemanuel Luna-Nemecio

Centro Universitario CIFE - México

josemanuelluna@cife.edu.mx

Karina X. Atayde-Manríquez

Universidad Autónoma del Estado de Morelos - México

karina.ataydeman@uaem.edu.mx

\section{RESUMEN}

Una significativa presencia de mujeres en el espacio universitario motiva la necesidad de investigar sobre la cultura de género en las universidades, máxime cuando persiste la discriminación e inequidad hacia las mujeres en estos espacios educativos. Para atender esta necesidad se realizó un análisis documental. El presente texto da cuenta de las líneas teóricas que auxilian en el análisis de la cultura de género de las universidades, los temas y las evidencias de la desigualdad de género. Las y los autores de los estudios revisados coinciden en la oportunidad invaluable que tienen estas comunidades como formadoras que cuestionen y renueven el modelo social y cultural de los estereotipos de género. Se proponen, asimismo, puntos de encuentro entre el enfoque socioformativo, en su eje del desarrollo social sostenible y la construcción de una cultura de equidad de género en los espacios universitarios.

Palabras clave: cultura de género; desigualdad; universidad; socioformación; desarrollo social sostenible.

\section{ABSTRACT}

A significant presence of women in university's spaces prompts the need to investigate gender culture in universities. Mainly, since discrimination and inequities still persist towards women in educational spaces. To assist this, need a documental analysis was done. This text expounds the different theoretical lines that help in the analysis of gender culture in universities, as well as, the subjects and evidence of gender inequality. The authors of the reviewed studies concur on the invaluable opportunity that this communities have as trainers that question and renew the social and cultural model of the gender stereotypes. Hence, an agreement is proposed between the socioformative focus, in its axis of sustainable social development and the construction of a culture of gender equality on university spaces.

Keywords: gender culture; inequality; university; socioformation; sustainable social development. 


\section{Introducción}

En las últimas dos décadas se ha verificado una presencia significativa de mujeres en los espacios universitarios, hecho que llama la atención pues desde los orígenes de la escuela moderna su composición había sido mayoritariamente masculina (Buquet, Cooper, Mingo \& Moreno, 2013). La educación había sido una opción negada para las mujeres. En la historia contemporánea sólo los hombres habían tenido este privilegio (Serret, 2008, citado en De Garay \& Del Valle-Díaz, 2012). Diferentes roles asignados a hombres y mujeres en la sociedad destinaban a las mujeres a tener un estatus social inferior que los hombres. El arribo de las mujeres a las universidades fue un largo proceso, propiciado por uno de los movimientos más importante del siglo XX: los movimientos feministas, que con sus reflexiones teóricas y activismo comenzaron a nombrar las diversas inequidades que viven las mujeres, destacando, entre otros temas, la exclusión que habían tenido en la educación (De Garay \& Del Valle, 2012). También una diversidad de ordenamientos jurídicos, políticas públicas de orden nacional o internacional han destacado las desigualdades, lo que llevó a la elaboración de lineamientos para propiciar el acceso de las mujeres a la educación.

En 1998, la UNESCO dio a conocer la Declaración mundial sobre la educación superior en el siglo XXI: Visión y acción, señalando como objetivo prioritario el fortalecimiento de la participación y promoción del acceso de las mujeres a espacios educativos (Montané \& Pessoa, 2012). De esta declaración mundial partió el interés de algunas instituciones por seguir este ordenamiento en México. El Instituto Nacional de las Mujeres (INMUJERES) y la Asociación Nacional de Universidades Institutos de Educación Superior (ANUIES) impulsaron la creación de la Red Nacional de Enlaces Académicos de Género en México, con la idea de promover la inclusión de la perspectiva de género en las universidades (Palomar, 2011). Asimismo, el 25 de septiembre de 2015, la Organización de las Naciones Unidas (ONU), aprobó la Agenda 2030 para el Desarrollo Sostenible que, entre uno de los 17 objetivos, se pretende alcanzar la igualdad entre los géneros y el empoderamiento de todas las mujeres y niñas. En su numeral 20 señala expresamente: "Las mujeres y las niñas deben tener igual acceso a una educación de calidad, a los recursos económicos y a la participación política, así como las mismas oportunidades que los hombres y los niños en el empleo, el liderazgo y la adopción de decisiones a todos los niveles" (ONU, 2015, p. 7). Es importante destacar la importancia de que dicha meta sea vista bajo la necesidad de replantear la caducidad que presentan los mencionados objetivos, bajo la imperante necesidad de definirlos bajo la perspectiva del desarrollo social sostenible (Luna-Nemecio, 2020).

En las reflexiones sobre el papel de las universidades en la actualidad, se coloca el énfasis en la trascendencia que tiene la educación para garantizar sociedades igualitarias y democráticas, además de la importancia que tienen en la producción, transmisión y divulgación de saberes y conocimiento, pero sobre todo, se hace alusión a la transformación y cambio que pueden propiciar una educación igualitaria en las y los estudiantes con nuevos discursos en las instituciones universitarias. Mas para proponer los contenidos de una educación equitativa, antes es necesario ¿conocer las particularidades de las relaciones de género de las universidades?, ¿qué desigualdades se presentan?, ¿cómo arribar al conocimiento de su cultura de género? (Buquet, et al., 2013; Palomar, 2005: Acuña-Rodríguez, 2014).

Al hablar de cultura de género en las universidades, nos referimos, al conjunto de pautas de pensamiento y acción, modos de actuar, regulados por repertorios de normas que se sustentan en un conjunto de valores que legitiman y hacen comprensibles comportamientos, creencias, símbolos, lenguajes, constituyendo un conjunto de prácticas, costumbres, hábitos naturalizados, legitimados e institucionalizados (García-Pérez, Rebollo, Vega, Barragán-Sánchez, Buzón \& Piedra, 2011). El género, como parte de la cultura, está presente en la vida de las instituciones y se expresa en todos los niveles de su funcionamiento. Es paradójico que, si bien los estudios de género surgieron en los espacios universitarios, fue hasta hace muy poco que las y los investigadores miraron hacia el interior de las comunidades universitarias para conocer la situación que guarda la igualdad de género en éstas (Ballarín, 2015; Díaz \& Anguita, 2017; Tomás, Castro \& Durán, 2012; Castillo \& Gamboa, 2013).

¿Dónde está?, ¿cómo se reproduce?, ¿quiénes sostienen la desigualdad en las universidades? ¿Cuál es el principio simbólico de ordenamiento social que establece un principio de diferencias, privilegios y jerarquías entre hombres y mujeres en los espacios educativos? El propósito de este estudio documental es dar respuesta a estas interrogantes, así como conocer las propuestas que se deslizan hacia el necesario cambio y transformación que requiere la universidad en donde prevalezca el respeto y la equidad (Buquet, et al., 2013, Páramo, 2010); y, en esta perspectiva, vincular el aporte que daría el enfoque educativo de la socioformación y del desarrollo social sostenible, que plantea la innovación de los procesos formativos a todos los niveles, centrándose en la gestión del talento humano y la resolución de los problemas del contexto, vinculando el pensamiento complejo. 
A partir de lo expuesto líneas antes, el estudio documental tiene las siguientes metas: 1) Definición del concepto de cultura de género en las universidades y líneas teóricas que auxilian en el diagnóstico de dicha cultura; 2) Sistematizar los aportes de las investigaciones sobre la cultura de género en las universidades; 3) Iniciar una reflexión sobre la importancia y los aportes que tendría la relación del enfoque socioformativo y el desarrollo social sostenible en la construcción de una cultura de equidad en las universidades.

\section{METODOLOGÍA \\ Tipo de Estudio}

El análisis documental llevado a cabo fue de tipo cualitativo (Rodríguez \& Luna-Nemecio, 2019), para conocer qué cultura de género existe en las universidades, formas de abordar las temáticas relativas y conocer el marco teórico utilizado y resultados de los estudios. En el presente texto se entiende por análisis documental la búsqueda y recuperación de información sustancial sobre el tema de la cultura de género en la universidad con el fin de reflexionar, procesar y seleccionar lo más pertinente hacia la solución de los objetivos de la investigación (Hernández-Ayala \& Tobón-Tobón, 2016). Se utilizaron tanto fuentes primarias como secundarias de investigadores e investigadoras que analizan y profundizan en algunos aspectos del tema. En su mayoría se trató de artículos de revistas, capítulos de libros, libros y normatividad nacional e internacional. Un rasgo del análisis documental consiste en presentar información de forma distinta a la original, generándose así un nuevo documento que enriquece el tema tratado (Peña \& Pirela, 2007) y en ese sentido se encuentra enfocado el presente análisis.

\section{Categorías de Análisis}

En el análisis documental sobre la cultura de género en las universidades, nos basamos en las siguientes categorías que permitieron la selección, organización y análisis de la información (ver Tabla 1).

Tabla 1. Categorías utilizadas en el estudio

\begin{tabular}{|l|l|l|}
\hline \multicolumn{1}{|c|}{ Categorías } & \multicolumn{1}{|c|}{ Subcategorías } & \multicolumn{1}{c|}{ Preguntas o componentes } \\
\hline $\begin{array}{l}\text { Cultura de género en la } \\
\text { Universidad }\end{array}$ & $\begin{array}{l}\text { Teoría de género } \\
\text { Sexismo } \\
\text { Discriminación } \\
\text { Estereotipos de género } \\
\text { Utilidad del concepto de } \\
\text { género } \\
\text { Equidad de género }\end{array}$ & $\begin{array}{l}\text { ¿Qué significa la cultura de género?, ¿Cómo conocer } \\
\text { la cultura de género en las universidades? } \\
\text { ¿Qué es el sexismo, la discriminación, los estereotipos } \\
\text { de género? } \\
\text { ¿Es útil el concepto de género? }\end{array}$ \\
\hline Perspectiva de género & $\begin{array}{l}\text { Metodologías para conocer } \\
\text { la cultura de género }\end{array}$ & $\begin{array}{l}\text { ¿Qué es la perspectiva de género?, ¿Quéimplicaciones } \\
\text { tiene en las universidades esta perspectiva? }\end{array}$ \\
\hline $\begin{array}{l}\text { La socioformación y la } \\
\text { cultura de equitativa de } \\
\text { género }\end{array}$ & Desarrollo social sostenible & $\begin{array}{l}\text { ¿Qué es la socioformación?, ¿Qué puntos de } \\
\text { encuentro tienen la socioformación y la cultura } \\
\text { equitativa de género? }\end{array}$ \\
\hline
\end{tabular}

Fuente: Elaboración propia.

En la presente indagación se tuvieron en cuenta los siguientes criterios para seleccionar los textos:

Se buscaron fuentes primarias y secundarias sobre el tema. Se llevó a cabo un análisis de textos académicos que abordaran aspectos relacionados con la "cultura de género en las universidades", que se ubicaron en las siguientes bases datos: Google Académico, Scopus, Scielo y Redalyc.

La selección se realizó a partir de las palabras clave: cultura de género y universidad; teoría de género; desigualdad de género, igualdad de género y universidad; género y docencia, de forma complementaria se utilizaron las palabras: "feminismo", "educación y género", "enfoque de género y universidad", "perspectiva de género y universidad".

Todos los artículos fueron publicados en el periodo de 2003 a 2017. 
También se revisaron libros importantes sobre teoría de género y desigualdad de género, que se ubican fuera del periodo señalado pero que eran de consulta indispensable.

Se utilizaron, asimismo, acuerdos y leyes nacionales e internacionales para fundamentar la influencia de dichos lineamientos en las indagaciones sobre la cultura de género en las universidades.

\section{Documentos Analizados}

En total se seleccionaron, analizaron y sistematizaron 55 textos (39 artículos, 12 libros y 3 lineamientos oficiales y 1 internacional), valorados de relevancia para el estudio y que cumplieron con los criterios señalados líneas antes, los cuales se describen en la Tabla 2.

Tabla 2. Documentos Analizados en el Estudio

\begin{tabular}{|l|c|c|c|}
\hline \multicolumn{1}{|c|}{ Documentos } & Sobre el tema & Latinoamericanos & Europa \\
\hline Artículos & 39 & 27 & 12 \\
\hline Libros & 13 & 9 & 3 \\
\hline $\begin{array}{l}\text { Lineamientos oficiales } \\
\text { México }\end{array}$ & 3 & & \\
\hline $\begin{array}{l}\text { Lineamientos } \\
\text { internacionales }\end{array}$ & 1 & & \\
\hline
\end{tabular}

Fuente: Elaboración propia.

\section{RESULTADOS}

\section{Cultura de Género en la universidad}

Se refiere a la serie de comportamientos, representaciones y prácticas que se presentan en una institución universitaria. Si en la mayoría de los estudios analizados se señala la existencia de desigualdad en las universidades, luego entonces, hay que saber en dónde está, cómo se produce y quiénes la sostienen (Buquet, et al., 2013; Palomar, 2011). También la cultura de género en la universidad es definida como red de signos, prácticas, tradiciones y costumbres, rutinas, rituales e inercias propias de una institución, donde se tejen los conflictos y negociación entre la diversidad de individualidades generando diversos juegos de poder (Acosta, 2012).

La forma de conocer esta cultura de género se ha realizado a través de investigaciones que diagnostican la manera como, mujeres y hombres, construyen y viven el género en la comunidad universitaria (académicos, administrativos y estudiantes), mostrar cómo se presenta la desigualdad, pues el género como principio simbólico de ordenamiento social conforma una estructura invisible y naturalizada, con efectos en todas las dimensiones de la vida cotidiana institucional (Palomar, 2011; Acosta, 2012; Piedra, García-Pérez, Fernández-García \& Rebollo, 2014). Los diagnósticos de género habrían de dar a conocer no sólo el mundo de las mujeres sino la situación del género, es decir, de mujeres y hombres.

\section{Teoría de género}

En la sociedad contemporánea ha persistido una mirada masculina en el mundo del conocimiento, sustentada en la supuesta inferioridad intelectual de las mujeres, por ello durante milenios las mujeres fueron excluidas de los ámbitos del saber. A partir de la segunda mitad del siglo XX, grupos de mujeres intelectuales y académicas construyeron un discurso propio que rompió con el discurso dominante sobre la feminidad y esa supuesta inferioridad. El concepto de género comenzó a utilizarse en las ciencias sociales a finales de los años 70, con el objetivo de diferenciar construcciones sociales y culturales con la biología (Lamas, 2000). Había que explorar cómo es que la diferencia sexual se convirtió en desigualdad entre hombres y mujeres.

El género es una herramienta teórica que permite mostrar la forma cómo, a partir de lo biológico, se asignan determinadas características apropiadas para las mujeres y los hombres, develándose la construcción social de la subordinación de la mujer y ciñéndola a ciertos espacios de actuación, que se pueden observar en el resultado de la producción de normas culturales, actitudes, sentimientos, valores, 
símbolos, conductas y actividades que la sociedad asigna y exige de forma diferente a la mujer y al hombre (Lamas, 2000; Lagarde, 1997; Palomar, 2011; Montané \& Pessoa, 2012; Durán, 2012; Trejo, Llaven \& Pérez, 2015; Mingo \& Moreno, 2017). Para Izquierdo (1998), la aproximación crítica a esta concepción no contempla la vida como un resultado, sino como un proceso, es decir, comprender a los sujetos construidos socialmente y, por tanto, condicionados, pero así mismo capaces de transformación.

Para algunos de los planteamientos feministas, el género sirve para visibilizar los papeles que tradicionalmente se ha asignado a las mujeres, presentándolas como sujetos pensantes y críticos (Palomar, 2011). La vinculación de género y feminismo tiene una dimensión política que busca una transformación para ellas y la sociedad en donde están inmersas.

Las posibilidades del concepto de género son variadas y dependen de los objetivos en función de políticas, economías y sociedades, ya sea para ámbitos educativos, familiares, políticos, los medios de comunicación, etc., es decir, depende del actor social que los utiliza, tales como políticos, autoridades, académicos, activistas sociales y ciudadanos (Palomar, 2011). Scott (2008) apunta que la utilización del concepto de género en la academia en los últimos 25 años ha sido en dos vertientes: el género referido únicamente a las mujeres y el género como construcción cultural de la diferenciación sexual, abordando las relaciones sociales de los sexos, mostrando que no hay un mundo de las mujeres aparte del mundo de los hombres, la información de las mujeres nos habla sobre los hombres (Scott, 2008). Para Palomar (2016), fueron las ciencias sociales las que convirtieron al concepto de género en una herramienta poderosa para explicar las desigualdades que se habían construido a partir de una diferenciación netamente sexual, utilizando al género como herramienta política y de acción, generando conceptualizaciones útiles como desigualdades sociales, violencias de género, conflictos de género y políticas de género.

A continuación, se definen otros conceptos que se derivan de la teoría de género y que sirven para develar las desigualdades de género que suceden de manera cotidiana y que precisan ser nombradas. Diversos feminismos y estudios de género han retomado la frase del filósofo francés Steiner (2013) quien aludiera a que lo que no se nombra no existe y es en ese sentido que la teoría de género ha buscado nombrar y con ello visibilizar situaciones y conceptos tales como: sexismo, discriminación y estereotipos de género.

\section{Sexismo}

Se define como una discriminación basada en el sexo de las personas, que beneficia a un sexo sobre otro, es decir, muestra a la mujer como un ser inferior a partir de sus diferencias biológicas con el hombre (INMUJERES, 2007). El sexismo afecta a hombres y mujeres, pues mientras las mujeres son educadas para estar en el ámbito privado de la casa y los hijos, a los hombres se les educa para ejercer el poder, en el ámbito público, todo ello representando desigualdad, pues las mujeres están limitadas a ejercer ciertas funciones sociales (Castillo \& Gamboa, 2013).

Los estudios de género también han mostrado los daños que se generan para los varones. De esta dimensión se han ocupado también los estudios conocidos como de las masculinidades (Figueroa, 2014, Salguero, 2013, Endara, 2018, Bonino, 1998) en los que se reflexiona sobre la situación de hombres y su malestar en los roles sociales y en donde se han generado también conceptos de utilidad como, por ejemplo, la masculinidad hegemónica referida por Kaufman (1995), o las precisiones en torno a los micromachismos (Bonino, 1995). Entre otras autoras y autores que se han interesando en estudiar el género desde diversas perspectivas.

Por todo lo antedicho, una educación no sexista implicaría la reestructuración de los programas de estudio de las currículas universitarias que eliminen los estereotipos de género, el lenguaje sexista (INMUJERES, 2007). También el sexismo es visto como un acto performativo que se presenta en el lenguaje y en los actos de comunicación como puestas en escena ritualizadas y que en las prácticas sociales legítima la condición de sujeción y subordinación de las mujeres, exhibiendo "el desprecio, el temor y el deseo que el sexo débil inspira" (Mingo \& Moreno, 2017).

\section{Discriminación}

En México la definición de discriminación se encuentra establecida legalmente en la Ley Federal para Prevenir y Eliminar la Discriminación que tiene como objetivo principal "promover la igualdad de oportunidades y de trato" y en su artículo $1^{\circ}$., inciso III la define como: "toda distinción, exclusión, restricción o preferencia que, por acción u omisión, con intención o sin ella, no sea objetiva, racional ni proporcional y tenga por objeto o resultado obstaculizar, restringir, impedir, menoscabar o anular el reconocimiento, goce o ejercicio de los derechos humanaos y libertades, cuando se base en uno o más de 
los siguientes motivos: el origen étnico o nacional, el color de la piel, la cultura, el sexo, el género, la edad, las discapacidades, la condición social, económica, de salud o jurídica, embarazo, lengua, religión, opiniones, preferencias sexuales, estado civil o cualquier otra, tenga por efecto impedir o anular el reconocimiento o el ejercicio de los derechos y la igualdad real de oportunidades de las personas (CNDH, 2018). Varios de estos motivos han sido utilizados para mostrar la inequidad de que han sido sujetas no sólo las mujeres (Trejo, Llaven \& Pérez, 2015), sino a la comunidad LGBTIQ y aliados, excluyéndoles y limitando su acceso a la equidad en todas las esferas de la vida social, económica, política, cultural y señalando las diversas formas de violencias en su contra.

\section{Estereotipos de género}

Se definen como las creencias, mitos y prejuicios que crean grupos humanos en una sociedad determinada, y son sociales en la medida que son compartidos por sus integrantes, es decir, invaden nuestra forma de pensar y señalan comportamientos esperados de mujeres y hombres (Palomar, 2011; Díaz de Greñu \& Anguita, 2017). Con frecuencia son despectivos respecto a la situación de las mujeres. Los estereotipos no son estables, pues se modifican de una sociedad a otra, así como en el tiempo y el espacio. A pesar de la presencia de las mujeres en los espacios educativos y su inserción en el trabajo remunerado, varios autores señalan la segregación horizontal que se escenifica en las universidades con la presencia de más mujeres en las disciplinas de las ciencias sociales y menos en las áreas científicas; también destacan que a partir de los estereotipos de género, con frecuencia profesores y profesoras, inconscientemente, tratan de distinta forma a mujeres y hombres en el aula (Piedra, García Pérez, Fernández-García \& Rebollo, 2014), aunque en el liderazgo hombres y mujeres tienen el mismo potencial, éste se sesga en los procesos estructurales, ya que "parte del contexto cultural, las expectativas y las preferencias de cada individuo para conformar los roles sociales de género" (De Garay, 2013, p. 287).

\section{Utilidad del concepto género}

En los años ochenta se presentan una serie de debates en torno al concepto de género, lo que ha producido una reflexión sobre su adecuada utilización (Izquierdo, 1998). Hay que destacar, que de los textos analizados para este estudio documental, únicamente abordan este debate los textos teóricos sobre el género, mientras que la mayoría utilizan el concepto de género en su acepción más conocida, aquella que se establece a partir del esquema sexo-género, destacando la utilidad del mismo, señalando que en la cultura actual en que vivimos nuestras actividades siguen codificadas como actividades masculinizadas y feminizadas, funcionando, también así, en las relaciones de género y poder. Para Bogino y Fernández-Rasines (2017), la crisis del concepto de género se presenta con la institucionalización de los estudios de género, pues éstos perdían su sentido crítico, por lo que dicho autor enfatiza la necesidad de seguir empleando el concepto de género para el análisis social, para interrogarnos cómo es que se establecen los significados diferenciados, qué implican y en qué contextos se presentan.

Para Palomar (2011), el concepto de género sigue vigente, pues hay poca consciencia en hombres y mujeres sobre la forma naturalizada de ser hombre o mujer culturalmente, no hay capacidad de darse cuenta de "esas reglas de una manera reflexiva y consciente, y menos aún de dar cuenta de lo que explicaría la existencia misma de esas reglas y no de otras, así como tampoco de la racionalidad de género que subyace en el orden institucional" (p. 30) y, si se da el caso, de que en hombres y mujeres haya un reconocimiento de esta situación o de su condición, hay resistencias al cambio.

\section{Equidad de género}

La equidad de género es un principio de justicia que reconoce la existencia de las diferencias sociales y la diversidad de necesidades de las personas, de los grupos y de los ambientes. En la equidad se incluyen dos ejes: el respeto y garantía de los derechos humanos y la igualdad de oportunidades para hombres y mujeres, en ese sentido las políticas de equidad no sólo se refieren al acceso al bienestar material, sino suponen cambios en la configuración socio-cultural de las relaciones de poder, lograr la justicia y la cohesión social (Glosario INMUJERES, 2007; Rebollo, Piedra de la Cuadra, Sala, Sabuco, Saavedra \& Bascón, 2012; Ordorika, 2015).

En el Artículo $6^{\circ}$. de la Ley General para Igualdad entre Mujeres y Hombres promulgado en México en el año 2006, se señala que igualdad de género se refiere a la eliminación de toda forma de discriminación en cualquiera de los ámbitos de la vida, que se originé por pertenecer a cualquier sexo. Toda política de equidad o de igualdad en las instituciones educativas necesita eliminar los estereotipos de género, acabar con los modelos de reproducción social y optar por modelos transformadores tomando en cuenta los comportamientos, aspiraciones y necesidades específicas de las mujeres y de los hombres, con un mismo valor y favorecidos de la misma manera (Ley General para la Igualdad entre Hombres y 
Mujeres, INMUJERES, 2007; Piedra, García-Pérez, Fernández-García \& Rebollo, 2014; lon, Duran-Bellonch \& Bernabeu, 2013).

\section{Perspectiva de género}

Uno de los aportes fundamentales de la teoría de género es la herramienta conceptual de la perspectiva de género, que se entiende como la mirada, el enfoque, la visión que permite analizar las características que definen a las mujeres y a los hombres de manera específica, así como sus semejanzas y diferencias, contribuyendo, de esta forma, a la "construcción subjetiva y social de una nueva configuración a partir de la resignificación de la historia, la sociedad, la cultura y la política desde las mujeres y con las mujeres" (Largarde, 1997, p. 13). Asimismo, es una acción para transformar los términos de las relaciones sociales y una voluntad política para alcanzar la equidad y la igualdad (Vargas, 2011; Durán, 2012). La utilización de la perspectiva de género permite nombrar de otra manera las cosas, mostrar los hechos ocultos dándoles otro significado. Los estudios de género comenzaron como un acto de revisión, proporcionaron un modelo del pensamiento, que permite la revisión y corrección consistente (Tomás \& Durán, 2012; Anguita, 2011).

Los estudios de género que comenzaron su institucionalización en 1980, parten de las concepciones tanto del género como de la perspectiva de género en sus reflexiones y sus aportaciones (Bogino \& Fernández-Rasines, 2017; Montané \& Pessoa, 2012; De Garay, 2013). La posibilidad de generación de nuevo conocimiento se presenta con la creación de centros, programas o institutos dedicados a los estudios de género en las universidades, así como la incorporación de dicha temática en los programas y planes de estudio, fortaleciendo de esta forma las herramientas teóricas y de transformación social (Buquet, 2011). La perspectiva de género exige de mujeres y hombres "cambios personales, íntimos y vitales, que no son aceptados por muchas personas que hoy usan el género como si fuera una herramienta técnica, neutra y edulcorable" (Lagarde, 1997, p. 21).

\section{Metodologías para conocer la cultura de género en las universidades}

Como se ha señalado, por la complejidad de las comunidades universitarias, es difícil utilizar una única propuesta de diagnóstico o análisis. El objetivo central de todas las investigaciones documentadas es la de evidenciar la existencia de inequidad de género en las universidades, a partir de metodologías cuantitativas (cuestionarios, datos estadísticos oficiales, pruebas ad hoc) (Piedra, García-Pérez, FernándezGarcía \& Rebollo, 2014; Palomar, 2011; Páramo, 2010) y cualitativas (entrevistas a profundidad, encuestas semiestructuradas, grupos de discusión, registro de anécdotas y observación sistemática, etnografía, estudios de caso) (Ríos, Mandiola \& Varas, 2017; Rebollo, Piedra de la Cuadra, Sala, Sabuco \& Saavedra, 2012), o ambas, generando instrumentos e indicadores para analizar temas específicos y en algún sector de la comunidad universitaria, por ejemplo, la invisibilidad de las mujeres en la academia y obstáculos para el desarrollo profesional, representaciones, códigos, roles y estereotipos de género en las y los docentes y en las y los estudiantes, segregación disciplinar, percepciones o actitudes hacia la igualdad, sexismo, etc. Y metodologías mixta para los estudios de toda la comunidad universitaria, por el tamaño y las diferencias que se pueden encontrar en las diversas instituciones de educación superior, la propuestas es llevar a cabo estudios que puedan arrojar una visión de la cultura de género de cada institución, a partir de una pregunta base: “¿cuál es la precisa forma de convivencia que, a partir del orden de género, se construye en el mundo universitario como parte de la cultura institucional de determinada universidad?" (Palomar, 2011: p. 13).

Entre los temas de los que parten las investigaciones sean cualitativas o cuantitativas revisadas, destacan:

La dominación masculina, como principio simbólico de ordenación social (Bogino \& FernándezRasines, 2017; Donoso, Montané \& Pessoa, 2014).

Feminización de la matrícula de las universidades (Buquet, et al, 2013; Ríos, Mandiola \& Varas, 2017). Algunas propuestas de políticas públicas sobre la educación y la igualdad de género (Acuña-Rodríguez, 2014; Bas, 2014).

La discriminación es deliberada e inconsciente, pues es encubierta por las costumbres y tradiciones de la comunidad estudiada (Palomar, 2011; García-Pérez, et al, 2011).

Uso de un lenguaje sexista (De Garay \& Del Valle, 2014; Tomás \& Durán, 2012).

Es importante señalar que el diagnóstico de la cultura de género en las universidades proporciona insumos necesarios para elaborar un diagnóstico y abrir el camino a una cultura institucional de equidad 
o igualdad de género (Palomar, 2011). Los resultados obtenidos en estas investigaciones han sido de gran relevancia y sientan los precedentes para subsiguientes estudios en otras universidades. Por ejemplo, de las investigaciones analizadas, se destaca que:

\begin{abstract}
1) Existe desigualdad a partir de observar que, en la actualidad, hay un mayor número de mujeres estudiando, pero aunque las mujeres han accedido a los estudios universitarios, sus oportunidades de crecimiento profesional y académico son muy limitadas en comparación con los hombres... (Ion, Duran-Bellonch \& Bernabeu, 2013; Tomás \& Durán 2012; De Garay, 2013; Acuña-Rodríguez, 2014).
\end{abstract}

2) La cultura organizativa de las universidades, que sigue siendo muy jerarquizada y dominada por los hombres, conlleva exclusión de las mujeres (Ion, Duran-Bellonch \& Bernabeu, 2013; De Garay, 2013).

3) En el desempeño académico, el sexo es un factor de gran incidencia. Aún cuando las mujeres enfrentan condiciones más adversas para estudiar (tales como: doble o triple jornada de estudio y trabajo, quehaceres domésticos, crianza de hijas e hijos u otros familiares), se gradúan en mayor cantidad que los hombres (Acuña-Rodríguez, 2014).

4) En el Informe Sombra para la Convención sobre la Eliminación de todas las formas de Discriminación contra la Mujer de los años 2007 a 2010 (Agenda Política de Mujeres, 2010), se menciona que: el sexismo y la discriminación siguen extendidos en el sistema educativo, pocas mujeres han ingresado a la educación técnica y al ámbito de la ciencia y tecnología, persistiendo brechas de género en el mercado laboral debido a la falta de estímulo para ingresar en campos tradicionalmente masculinos, con frecuencias, las mujeres reciben salarios menores que los hombres por el mismo tipo de trabajo. Pocas mujeres se encuentran en puestos de toma de decisiones dentro de su campo de trabajo (Acuña-Rodríguez, 2014; De Garay, 2013).

Algunos estudios se auxilian de otros conceptos de las ciencias sociales en aras de enriquecer los resultados de sus investigaciones. Por ejemplo, a partir de ver a la escuela como un microcosmos social, concepto tomado de la sociología, se señala que el género, aprendido socialmente e inseparable del sexo, está presente en los programas educativos, en los libros de texto, en el lenguaje cotidiano; en el currículo oficial oculto, está todo aquello que está regido por una ideología sexista que promueve la desigualdad (Vargas, 2011; Díaz de Greñu \& Anguita, 2017). También, por ejemplo, a partir de evaluar las buenas prácticas coeducativas en los centros educativos de Andalucía, España, enmarcados en el Plan de Igualdad estatal, se propuso la creación de un responsable de la igualdad con el objetivo de corregir los desequilibrios derivados de roles de género y promover la inclusión (Rebollo, Piedra, Sala, Sabuco, Saavedra \& Bascón, 2012).

Al tomar el género como un elemento para evaluar la calidad de la educación, se evidencias las profundas problemáticas y dificultades de las mujeres en las universidades, como el famoso "techo de cristal", la "cortina de humo", la "pared de la maternidad", las expectativas frustradas, el acoso sexual, la invisibilidad ante los colegas masculinos, las dificultades de conciliación familia-trabajo, los procesos de exclusión en la promoción profesional, etc. (De Garay, 2013; Donoso, Montané \& Pessoa, 2014; LópezFrancés, Viana-Orta \& Sánchez-Sánchez, 2016).

Se analiza el currículo oculto, visto como el conjunto de ideas, prejuicios, experiencias, etc., que sin darse cuenta se transmite a las y los estudiantes, a pesar de contar con un currículo oficial se ofertan otras ideas (Díaz de Greñu \& Anguita, 2017). A partir de indicadores sobre la percepción de desigualdades de género en la universidad, tomando como base el reconocimiento académico en forma de publicaciones y dirección de proyectos, la presencia en medios de comunicación y la presencia en diversos ámbitos de la actividad académica: docencia, investigación y gestión (Ion, Duran-Bellonch \& Bernabeu, 2013; Tomás \& Durán, 2012). A partir de una metodología cuantitativa centran su diagnóstico en conocer las actitudes e ideas sobre el género o la igualdad de género (Piedra, García-Pérez, Fernández-García \& Rebollo, 2014).

\title{
La socioformación y el concepto de género
}

La socioformación es una propuesta educativa creada colectivamente por investigadoras e investigadores de América Latina cuyo objetivo es transformar la educación tradicional, ilustrada y moderna, a partir del enfoque socioformativo, que propone trascender la educación basada en contenidos y desapegada del entorno social, político, económico y cultural. El enfoque socioformativo propone formar personas integrales preparadas para afrontar los retos actuales de la sociedad del conocimiento, a partir de contar con un sólido proyecto ético de vida, trabajo colaborativo, emprendimiento y gestión del 
conocimiento y utilizando las tecnologías de la información y la comunicación (Tobón, 2015). Las metas de la socioformación son: tener calidad de vida, convivencia, desarrollo económico y sustentabilidad (LunaNemecio, 2020).

El enfoque socioformativo es una propuesta en construcción que propone vincular la educación de cada institución con la formación integral y la responsabilidad, con un currículo socioformativo flexible orientado en la resolución de problemas de contexto. Para este enfoque es sustancial formar a los estudiantes en 6 competencias: 1) Proyecto ético de vida; 2) Emprendimiento; 3) Trabajo colaborativo; 4) Gestión del conocimiento; 5) Desarrollo social sostenible y 6) Comunicación bilingüe

\section{La socioformación y la cultura de la equidad de género}

Un punto de encuentro entre la socioformación y la cultura de equidad de género es su marco de reflexión-acción, pues ambas visiones plantean una transformación del modelo tradicional de educación. De los aportes de la teoría del género está la propuesta de la perspectiva de género. Para Lagarde (1997): "una humanidad diversa y democrática requiere que mujeres y hombres seamos diferentes de quienes hemos sido, para ser reconocidos en la diversidad y vivir en la democracia genérica" (p. 13). El enfoque socioformativo promueve una formación humana integral con un proyecto ético de vida, aprender a emprender y vivencia cultural, en donde, desde mi punto de vista, está incluida la equidad entre hombres y mujeres. Tobón (2013) señala que desde la socioformación se estudia al ser humano como es, pero, sobre todo, lo que puede llegar a hacer de forma constructiva y ética, con miras al fortalecimiento del tejido social y el desarrollo económico.

Si bien aún no hay estudios que nos permitan afinar las relaciones entre la equidad de género y la socioformación podemos comenzar a delinear en algunos puntos de encuentro:

Construir un proyecto ético de vida significa comprometerse con la equidad entre mujeres y hombres en todos los ámbitos de la institución educativa.

Aprender a emprender con perspectiva de género, implica desarrollar proyectos que incluyan el conocimiento, la creatividad e imaginación de hombres y mujeres.

La vivencia cultural tiene que caminar hacia la desaparición de los estereotipos de género, pues hombres y mujeres tienen el mismo potencial.

El trabajo colaborativo debe estar sustentado en relaciones de igualdad entre hombres y mujeres.

Un desarrollo social sostenible implica también la forma en que las mujeres y hombres participan, se benefician y administran los recursos de los proyectos y actividades de manera diferenciada, es decir, se transfiere la atención sobre las mujeres como grupo potenciador del desarrollo en igualdad de oportunidades que los hombres, manteniendo una relación armónica y en equilibrio con la naturaleza.

Otra posibilidad de confluencia la podemos observar en la propuesta de la formación desde la socioformación, ya que ésta "da cuenta de la integración de las dinámicas sociales y contextuales que operan sobre el sujeto con las dinámicas personales; por ello la formación es la resultante de la articulación de los procesos sociohistóricos y procesos individuales, a través del lenguaje y la comunicación" (Tobón, 2013, p. 47). Lo anterior se relaciona con las diferentes propuestas de los artículos analizados, en el sentido de que la educación se ha convertido en clave para la promoción y la inclusión social de todos y todas; se señala que es necesarios el acceso a nuevas habilidades y competencias para hacer frente de forma crítica a los rápidos cambios sociales que se están produciendo día a día en la sociedad del conocimiento (Elboj y Oliver, 2003).

La socioformación señala que el diálogo, la comunicación e interacción entre los diferentes miembros y agentes de una comunidad pasarán a ser características predominantes del aprendizaje, pero no sólo en el aula, pues tendrán un papel esencial, la calle y la casa, luego entonces, para Elboj y Oliver (2003), las escuelas tendrán que convertirse en comunidades de aprendizaje. Desde la cultura de equidad de género se propone superar la desigualdad y exclusión de las mujeres como una estrategia para llegar a un desarrollo sostenible en las sociedades (Buquet, 2011), donde la creación de saberes da la posibilidad de alcanzar el horizonte del desarrollo social sostenible (Luna-Nemecio, 2019).

El desarrollo social sostenible es una de las 6 competencias que habrá que desarrollarse en los ámbitos escolares, para del beneficio de los y las estudiantes, su familia, su comunidad, reto superior que tienen las universidades latinoamericanas con sociedades donde la exclusión y la desigualdad son muy 
visibles y permanentes. El enfoque socioformativo es innovador pues plantea formar a partir de problemas reales como la pobreza, la salud, la contaminación, escases de recursos, la desigualdad, la exclusión, con actividades participativas que tienen el objetivo de lograr una formación integral de ciudadanos y ciudadanas, desarrollando el talento humano mediante la gestión de proyectos que busquen la mejora constante de la vida, de la comunidad a través de la colaboración (Izquierdo-Merlo, 2020).

Como parte del desarrollo social sostenible desde el enfoque de la socioformación están la gestión del conocimiento, la mejora de datos que guíen la acción de las comunidades educativas, con un contenido ético y humano, donde está implícita la equidad de género. Un desarrollo social sostenible abordaría la redistribución equitativa de las actividades entre los sexos, en lo público y lo privado; la justa valoración de los distintos trabajos que realizan mujeres y hombres, especialmente en lo referente a la crianza de las hijas e hijos, el cuidado de los enfermos y las tareas domésticas; así como la modificación de las estructuras sociales, los mecanismos, reglas, prácticas y valores que reproducen desigualdad.

La socioformación plantea la conformación de un currículo flexible orientado en la resolución de problemas de contexto, en éste podría integrarse la perspectiva de género, es decir, incorporar en la discusión en clase, en la casa, en la calle, los temas con perspectiva de género, ya sean reflexiones de las diferencias y diversidad de identidades, o el cuestionamiento de los estereotipos sexistas, o el papel de las mujeres en la historia, también reflexionar cómo desaprender la violencia y la discriminación hacia las mujeres, todo ello aportará a la formación de las y los jóvenes universitarios elementos para la deconstrucción de las diversas formas de discriminación imperantes en nuestras sociedades y les transmite valores de equidad y respeto a las diferencias. Pues, como afirma Tobón (2013): "mediante la autorreflexión, cada persona siempre tiene la posibilidad de construir su forma de ser, pensar y sentir, tomando así distancia con las imposiciones y bloqueos que con frecuencia el contexto social impone" (p. 35).

\section{DISCUSIÓN}

A partir de la revisión documental realizada podemos afirmar que en los espacios universitarios se han reproducido las prácticas de desigualdad entre los géneros, naturalizándose ésta, así como las relaciones de dominación del hombre sobre la mujer (Ríos, Mandiola \& Varas, 2017; Mingo, 2016; LópezFrancés, Viana-Orta \& Sánchez-Sánchez, 2016; Acuña-Rodríguez, 2014; Durán, 2012; Páramo, 2010). LoS rasgos de esta desigualdad se han presentado a lo largo de este estudio documental. La escuela, como uno de los pilares de sociedad, ha sido la reproductora de la cultura de desigualdad, pues en ésta se articula, fomenta y reproduce el orden social, económico y político, en sus espacios se da la naturalización de las desigualdades y de las relaciones de dominación (Vargas, 2011; Páramo, 2010; Acuña-Rodríguez, 2014).

Un segundo aspecto a destacar es que son pocos los cambios estructurales y las políticas de las instituciones universitarias para resolver la desigualdad, muchas de sus acciones han quedado sólo en discursos y en cambios administrativos y de gestión. La introducción de la perspectiva de género en los espacios universitarios no debería ser sólo discursiva, sino tener efectos y evidencias en todos los planos de la cultura institucional: vida cotidiana, en los rituales, en las formas de organización, en las políticas, en los reglamentos, en los símbolos, en los programas docentes y de investigación (Palomar, 2011; Ordorika, 2015; Acosta, 2012). La instauración de la perspectiva de género dotaría de esa particular mirada para comprender la vida social, la manera como ser hombre o mujer es un dato cultural y no biológico, y como la forma que presentan las desigualdades sociales basadas en el sexo de las personas está relacionado con la manera como se construye la oposición hombre/mujer en el imaginario social (Palomar, 2011).

También hay que apuntar que en México no se tienen políticas o instrumentos jurídicos suficientes y efectivos que exijan a las universidades asegurar la igualdad de género en todos los ámbitos universitarios, que tengan instancias y normatividad específicas que vigilen y sancionen cualquier acto de discriminación y/o violencia de género, como si lo tienen, por ejemplo, los partidos políticos para asegurar la paridad entre hombres y mujeres en los cargos de elección popular. Un logro importante en algunas universidades ha sido la creación de espacios (unidades, secretarías o centros) para desarrollar estudios de género y en algunas universidades se ha avanzado en la elaboración de protocolos contra la violencia hacia las mujeres.

Otro dato importante es que para arribar a una equidad de género en las universidades se requiere de un proceso de aculturación que cambie concepciones y prácticas socialmente aprendidas. En algunos de los estudios analizados, se hace hincapié en la formación docente, es decir, que sobre todo las y los profesores tengan una educación en género (Castillo \& Gamboa, 2013; Bas, 2014; López-Francés, VianaOrta \& Sánchez-Sánchez, 2016). Para otros investigadores e investigadoras, la sensibilización y formación en género son aspectos importantes en la innovación educativa (García-Pérez, et al., 2011); se requieren competencias docentes de "saber hacer", pero un saber que se aplique de forma reflexiva, con carácter 
integrador, abarcando conocimientos, procedimientos, emociones, valores y actitudes en la integración de la perspectiva de género.

Las aportaciones intelectuales de las mujeres tienen que ser incorporadas en el currículo oficial de las escuelas, por ejemplo, la educación afectivo-sexual y el aprendizaje del manejo de las actividades reproductivas (cuidado de la infancia y de los ancianos) y el conocimiento de auto-suficiencia y de responsabilidad familiar (Anguita, 2011; Araya, 2014; Díaz y Anguita, 2017), es impostergable que la perspectiva de género sea parte los diversos currículos universitarios (Piedra, García-Pérez, FernándezGarcía \& Rebollo, 2014; Anguita, 2011).

Hay que señalar que, si el reto de las comunidades universitarias es superar la inequidad, es necesario profundizar en la investigación para conocer las relaciones de género imperantes en las comunidades y corregirlas. Si no cuentan con instrumentos modelos que diagnostiquen la desigualdad, entonces, cada institución de acuerdo a particularidades, contextos y necesidades, debe desarrollar el propio para conocer cómo, quién, dónde se produce y reproduce la desigualdad (Palomar, 2011). Romper el "techo de cristal" implica que las mujeres participen en las decisiones de las políticas institucionales universitarias, asumiendo cargos directivos. Para que las mujeres habiten las universidades se requiere que hagan propios los espacios, se sientan seguras, que los vivan sintiendo que son esenciales para dirigirlos y transformarlos, y para ello se requiere poder (Ballarín, 2015). Finalmente, construir una cultura de equidad de género en las universidades es llevar la propuesta ética que es la base de los estudios de las mujeres y de género al plano institucional (Buquet, 2011; Palomar, 2011, Ion, Duran-Bellonch \& Bernabeu, 2013).

Queda pendiente profundizar en la relación y puntos de confluencia que tendrían la perspectiva de género y el enfoque socioformativo, por ejemplo, cómo retroalimentar ambas perspectivas para potenciar su acción-reflexión que transformen las instituciones educativas en ambientas más igualitarios y acordes a las necesidades que presenta la sociedad del conocimiento.

\section{Conflict of interest}

No potential conflict of interest is reported by the author(s).

\section{Funding}

There is no financial assistance in studies from external parties.

\section{Acknowledgements}

Reconocemos a la Dra. María Guadalupe Veytia Bucheli y al Dr. Sergio Raúl Herrera Meza del Centro Universitario CIFE por los comentarios y apoyo prestado para el diseño de la presente investigación.

\section{REFERENCIAS}

Acosta, A. (2012). La cultura institucional de género en la Universidad de Guadalajara. Revista de la Educación Superior, 4 (164), 149-155. https://www.redalyc.org/pdf/604/60426901007.pdf

Acuña-Rodríguez, M. C. (2014). Reflexiones en torno al vínculo género educación superior. Educación, 38 (2), 89-106. Recuperado de http://www.redalyc.org/articulo.oa?id=44031370006

Anguita, R. (2011). El reto de la formación del profesorado para la igualdad. Revista Electrónica Interuniversitaria de Formación del Profesorado, 14 (1), 43-51. Recuperado de http://www.redalyc.org/articulo.oa?id=217017192004

Araya, S. (2014). La categoría analítica de género: notas para un debate. Hallazgos, 12(23), 287-305. https://doi. org/10.15332/s1794-3841.2015.0023.014.

Ballarín, P. (2015). Los códigos de género en la universidad. Revista Iberoamericana en Educación, (68), 19-38. https:// doi.org/10.35362/rie680168

Bas, E. (2014). Educación social y género Pedagogía Social. Revista Interuniversitaria, (23), 13-20. https://recyt.fecyt.es/ index.php/PSRI/article/view/37080/20627

Bogino, M., y Fernández-Rasines, P. (2017). Relecturas de género: concepto normativo y categoría crítica. Revista de estudios de género, La ventana, (45), 158-185. https://doi.org/10.32870/lv.v5i45.5375

Bonino L. (1995). Los micromachismos en la vida conyugal. En Corsi, J., Violencia masculina en la pareja. Buenos Aires: Paidós.

Bonino, L. (1998). Desconstruyendo la “normalidad” masculina. Actualidad Psicológica. (254), 25-27.

Buquet, A. (2011). Transversalización de la perspectiva de género en la educación superior. Problemas conceptuales prácticos. Revista Perfiles educativos, (33). http://www.scielo.org.mx/pdf/peredu/v33nspe/v33nspea18.pdf 
Buquet, A., Cooper J., Mingo A., \& Moreno, H. (2013). Intrusas en la Universidad. México. UNAM, PUEG, Instituto de Investigaciones sobre la Universidad y la educación.

Castillo, M., \& Gamboa, R. (2013). La vinculación de la educación y género. Revista Actualidades Investigativas en Educación, 13(1), 1-16. http://www.redalyc.org/articulo.oa?id=44725654014

Comisión Nacional de los Derechos Humanos (CNDH). (2018). Ley Federal para prevenir y eliminar la discriminación. Última reforma publicada en el Diario oficial de la Federación el 21 de junio de 2018.

De Garay, A., y Del Valle-Díaz, M. (2012). Una mirada a la presencia de las mujeres en la educación superior. Revista Iberoamericana de Educación Superior, 3(6), 3-30. http://ries.universia.net

De Garay, A. (2013). Igualdad de Género en la Universidad Autónoma Metropolitana: un espejismo. Revista La Ventana. 4(38). 277-330. https://doi.org/10.32870/lv.v4i38.534

Díaz de Greñu, S., y Anguita, R. (2017). Estereotipos del profesorado en torno al género y la orientación sexual. Revista Electrónica Interuniversitaria de Formación del Profesorado, 20(1), 219-232. https://dx.doi.org/10.6018/ reifop.20.1.228961

Donoso-Vázquez, T, Montané, A., \& Pessoa, M. E. (2014). Género y calidad en Educación Superior. Revista Electrónica Interuniversitaria de Formación del Profesorado, 17 (3), 157-171. https://dx.doi.org/10.6018/reifop.17.3.204121

Durán, M. (2012). La Transversalidad de Género en la Educación Superior: propuesta de un modelo de implementación. Revista Posgrado y Sociedad. Sistema de Estudios de Posgrado, 12(1), 23-43. https://doi.org/10.22458/rpys.v12i1.1701

Elboj, C. y Oliver, E. (2003). Las comunidades de aprendizaje: Un modelo de educación dialógica en la sociedad del Conocimiento. Revista Interuniversitaria de Formación del Profesorado, 17(3), 91-103. http://www.redalyc.org/ articulo.oa?id=27417306

Endara, G. (2018) (editor). ¿Qué hacemos con las masculinidades? Reflexiones antipatriarcales para pensar del privilegio al cuidado. Ecuador: Friedrich Ebert Stifun.

Figueroa, J.G. (2014) (coordinador). Políticas públicas y la experiencia del ser hombre. Paternidad, espacios laborales, salud y educación. México: El Colegio de México.

García-Pérez, R., Rebollo, M. A., et al. (2011). El patriarcado no es transparente: competencias del profesorado para reconocer desigualdad. Revista Cultura y Educación, 23(3), 385-397. http://doi.org/10.1174/113564011797330298

Hernández-Ayala, H., y Tobón-Tobón, S. (2016). Análisis documental del proceso de inclusión en la educación. $R a$ Ximhai, 12(6), 399-420. https://www.redalyc.org/pdf/461/46148194028.pdf

Instituto Nacional de las Mujeres. (2007). Glosario de Género. México.

Instituto Nacional de las Mujeres. (2007). Ley General para la Igualdad entre Hombres y Mujeres. México.

Ion, G., Duran-Bellonch., y Bernabeu, M. D. (2013). El profesorado y su percepción sobre la igualdad de género en la universidad. Revista Complutense de Educación, 24(1), 123-140. http://dx.doi.org/10.5209/rev_RCED.2013.v24. n1.41194

Izquierdo, M. (1998). El malestar en la desigualdad. España: Ediciones Cátedra, Universitat de València, Instituto de la Mujer.

Izquierdo-Merlo, M. E. (2020). Knowledge management from the socioformative approach towards sustainable social development. Ecocience International Journal, 2 (2), 10-18. http://doi.org/10.35766/je20222

Kaufman M. (1994). Men, Feminism, and Men's Contradictory Experiences of Power. En, Harry Brod y Michael Kaufman (editores). Theorizing Masculinities. Thousand Oaks, CA. Sage Publications.

Lagarde, M. (1997). Género y feminismo. España: Editorial horas y horas.

Lamas, M. (2000). Usos, dificultades y posibilidades de la categoría “género". El género. La construcción cultural de la diferencia sexual. México: PUEG, Grupo Editorial Miguel Ángel Porrúa.

López-Francés, I., Viana-Orta, M., y Sánchez-Sánchez, B. (2016). La equidad de género en el ámbito universitario: ¿un reto resuelto? Revista Electrónica Interuniversitaria de Formación del Profesorado, 19(2), 349-361. http://dx.doi. org/10.6018/reifop.19.2.211531.

Luna-Nemecio, J. (2020). Para pensar el desarrollo social sostenible: múltiples enfoques, un mismo objetivo. Mount Dora (USA)/Quito (Ecuador): Kresearch/Religación. Centro de Investigaciones en Ciencias Sociales y Humanidades desde América Latina. Doi: https://doi.org/10.35766/dss20

Mingo, A. (2016). 'iPasen a borrar el pizarrón!’ Mujeres en la universidad. Revista de Educación Superior 45(178), 1-15, México, ANUIES. http://dx.doi.org/10.1016/j.resu.2016.03.001

Mingo, A., y Moreno, H. (2017). Sexismo en la Universidad. Estudios Sociológicos, 35(105). 575-595. https://doi. org/10.24201/es.2017V35n105.1434

Montané, A., y Pessoa de Carvalho, M. (2012). Diálogo sobre género: justicia, equidad y políticas de igualdad en 
educación superior (Brasil y España). Revista Lusófona de Educação, (21), 97-120. https://revistas.ulusofona.pt/ index.php/rleducacao/article/view/3083

Ordorika, I. (2015). Equidad de género en la Educación Superior. Revista de la Educación Superior, 2 (174), 7-17. Recuperado de http://www.redalyc.org/articulo.oa?id=60439229001

Organización de las Naciones Unidas (ONU) (2015). Agenda 2030 para el Desarrollo Sostenible. Consultado Recuperado de https://bit.ly/35fGkzk

Palomar, C. (2005). La política de género en la educación superior. Revista de Estudios de Género. La ventana, (21), 7-43, Recuperado de http://www.redalyc.org/articulo.oa?id=88402101

Palomar, C. (2011). La cultura institucional de género en la Universidad de Guadalajara. México: Asociación Nacional de Universidades e Instituciones de Educación Superior.

Palomar, C. (2015). El vacío del género. Revista Asparkía, (26), 17-33.

Palomar, C. (2016). Veinte años de pensar el género. Debate Feminista, (52), 34-49.

Páramo, P. (2010). Las representaciones de género en profesores universitarios. Estudios Pedagógicos, 36(2), 177-193, Recuperado de http://www.redalyc.org/articulo.oa?id=173518942009

Peña, T. y Pirella, J. (2007). La complejidad del análisis documental. Información, cultura y sociedad: revista del Instituto de Investigaciones Bibliotecológicas, (16), 55-81. http://revistascientificas.filo.uba.ar/index.php/ICS/article/view/869

Piedra, J., García-Pérez, R., Fernández-García, E., y Rebollo, M. (2014). Brecha de género en educación física: actitudes del profesorado hacia la igualdad. Revista Internacional de Medicina y Ciencias de la Actividad Física y el Deporte, 14 (53), 1-21. http://cdeporte.rediris.es/revista/revista53/artbrecha438.htm

Rebollo, C., Piedra de la Cuadra, J., Sala, A., et al. (2012). La equidad de género en educación: análisis y descripción de buenas prácticas educativas. Revista de Educación, (38), 129-152. https://doi.org/10.4438/1988-592XRE-2012-358-186

Ríos, N., Mandiola, M., y Varas, A. (2017). Haciendo género, haciendo academia: Un análisis feminista de la organización del trabajo académico en Chile. Psicoperspectivas. Individuo y Sociedad, 16(2), 114-124. https://doi.org/10.5027/ psicoperspectivas-vol16-issue2-fulltext

Rodríguez, O., y Luna-Nemecio, J. (2019). Educación musical para el desarrollo sostenible: una revisión documental. Revista da abem. Associaçap brasileira de edicaçao musical, 27(43), 132-149.

Salguero, A. (2013). Masculinidad como configuración dinámica de identidades. En, Juan Carlos Ramírez y José Carlos Cervantes (coord.), Los hombres en México. Veredas recorridas y por andar. Una mirada a los estudios de género de los hombres, las masculinidades (pp. 37-52). México: Universidad de Guadalajara.

Scott, J. W. (2008). Género e historia. México: Fondo de Cultura Económica, Universidad Autónoma de la Ciudad de México.

Steiner, G. (2013). Lenguaje y silencio: ensayos sobre la literatura, el lenguaje y lo inhumano. España: Editorial Gedisa.

Tobón, S. (2015-2016). La socioformación: avances y retos en la sociedad del conocimiento. Conferencias Magistrales del XIII Congreso Nacional de Investigación Educativa. Consejo Mexicano de Investigación Educativa.

Tobón, S (2013). Formación integral y competencias: pensamiento complejo, currículo, didáctica y evaluación. $4^{\mathrm{a}}$. Ed. Bogotá: Ecoe Ediciones.

Tomás, M., Castro, D., \& Durán, M. (2012). Aproximación a un modelo de análisis de la visibilidad en la universidad desde la perspectiva de género. Bordón 64(1), 141-155. https://recyt.fecyt.es/index.php/BORDON/article/ view/21983

Trejo, M. L., Llaven, G., y Pérez, H. (2015). El enfoque de género en la educación. Atenas, 4(32), 49-61. https://www. redalyc.org/pdf/4780/478047208004.pdf

Vargas, I. (2011). Reflexiones en torno a la vinculación de la Educación y el género. Revista Electrónica Educare, 15(1), 137-147. http://www.redalyc.org/articulo.oa?id=194118804011

\section{Authors:}

Ana María Hernández-López. Doctorante en Socioformación y Sociedad del Conocimiento (Centro Universitario CIFE). Profesora-investigadora en la Universidad Autónoma de la Ciudad de México

Josemanuel Luna-Nemecio. Doctor en Geografía por la Universidad Nacional Autónoma de México. Profesor Investigador en el Centro Universitario CIFE (México). Investigador en Ekap University (Estados Unidos).

Karina X. Atayde-Manríquez. Candidata a Doctora en Ciencias Sociales por la Universidad Autónoma del Estado de Morelos. 